\title{
CARACTERÍSTICAS REPRODUCTIVAS Y CALIDAD DE SEMILLA EN POBLACIONES FRAGMENTADAS DE Pinus chiapensis (MARTÍNEZ) ANDRESEN
}

\author{
REPRODUCTIVE CHARACTERISTICS AND SEED QUALITY IN FRAGMENTED \\ POPULATIONS OF Pinus chiapensis (MARTÍNEZ) ANDRESEN
}

\author{
Elisea Capilla-Dinorin ${ }^{1}$, Javier López-Upton ${ }^{1 *}$, \\ Marcos Jiménez-Casas ${ }^{1}$ y Virginia Rebolledo-Camacho ${ }^{2}$
}

\begin{abstract}
'Colegio de Postgraduados, Postgrado en Ciencias Forestales, Montecillo, Estado de México, México. ${ }^{2}$ Universidad Veracruzana, Instituto de Investigaciones Forestales, Xalapa, Veracruz, México.

*Autor de correspondencia (jlopezupton@gmail.com)
\end{abstract}

\section{RESUMEN}

Pinus chiapensis (Martínez) Andresen es un árbol de rápido crecimiento, apreciado por su calidad de madera, cuyas poblaciones septentrionales en los límites estatales de Puebla y Veracruz, México son pequeñas, están fragmentadas y presentan repoblación reducida. El objetivo del presente estudio fue caracterizar algunos rasgos reproductivos asociados con la regeneración de siete poblaciones de Pinus chiapensis en dichos estados. Se muestreó un total de 125 árboles (de 15 a 22 árboles por población) y se evaluó la semilla cosechade de una muestra de 10 conos maduros por árbol. Se determinó el largo, ancho y peso seco de cono, el potencial de producción de semilla, los porcentajes de semilla llena, vana, plagada y óvulos abortados, además del peso promedio de la semilla. Se realizó una prueba de germinación en una cámara de ambiente controlado. En todas variables evaluadas se detectaron diferencias significativas entre poblaciones. El largo y ancho promedio de cono fue de 95.9 y $28.7 \mathrm{~mm}$, respectivamente; ambas correlacionaron positivamente con la altitud de origen de la población. El número potencial promedio de semillas por estróbilo fue de 89 , de las cuales resultaron 59 \% óvulos abortados, $22.6 \%$ semillas vanas, $0.9 \%$ semillas plagadas y $17.5 \%$ semillas llenas, lo que indica una reducida eficiencia de producción de semilla (1 a 16 semillas llenas por cono). El alto porcentaje de óvulos abortados indica problemas de polinización asociados con el tamaño reducido de las poblaciones. Se encontró una eficiencia reproductiva de $25.6 \mathrm{mg}$ de semilla por gramo de cono. La semilla mostró una capacidad germinativa de $75.7 \%$, un valor pico de $3.74 \%$ por día y un valor germinativo de 9.74. A pesar de la baja producción de semillas llenas y el alto porcentaje de semillas vanas, las poblaciones evaluadas produjeron semillas llenas de aceptable calidad germinativa.

Palabras clave: Pinus chiapensis, características reproductivas, especies en riesgo, variación morfológica.

\section{SUMMARY}

Pinus chiapensis (Martínez) Andresen is a fast-growing tree, appreciated for its wood quality, whose northern populations, in the state limits of Puebla and Veracruz, Mexico are small, fragmented and with reduced regeneration. The objective of this study was to characterize some reproductive traits associated with regeneration of seven Pinus chiapensis populations in these states. A total of 125 trees were sampled ( 15 to 22 trees per population) and the seed harvested from a sample of 10 mature cones per tree was evaluated. The length, width and dry weight of the cones, the potential for seed production, percentages of filled, empty and plagued seeds, aborted ovules and the average weight of the seed were determined. A germination test was carried out in a controlled-environment chamber. Significant differences were detected between populations in all variables evaluated. The average cone length and width were 95.9 and $28.7 \mathrm{~mm}$, respectively; both positively correlated with the altitude of origin of the population. The average potential number of seeds per strobilus was 89 , of which $59.0 \%$ were aborted ovules, $22.6 \%$ empty seeds $0.9 \%$ plagued seeds and $17.5 \%$ filled seeds, which indicates a low efficiency of seed production (1 to 16 filled seeds per cone). The high percentage of aborted ovules indicates pollination problems associated with the small size of the populations. A reproductive efficiency of $25.6 \mathrm{mg}$ of seed per gram of strobilus was found. The seed showed a germination capacity of $75.7 \%$, a peak value of $3.74 \%$ per day and a germinative value of 9.74 . Despite the low production of filled seeds and the high percentage of empty seeds, the evaluated populations produce filled seeds of acceptable germination quality.

Index words: Pinus chiapensis, endangered species, morphological variation, reproductive characteristics.

\section{INTRODUCCIÓN}

Pinus chiapensis (Martínez) Andresen es un recurso forestal importante del bosque nublado de México y norte de Guatemala (Del Castillo y Acosta, 2002). Es un árbol apreciado por su alta tasa de crecimiento y calidad de madera; sin embargo, el estado de conservación de $P$. chiapensis es preocupante debido a la fragmentación de sus poblaciones asociada con el cambio de uso del suelo y la explotación de este árbol como recurso maderero (Del Castillo y Acosta, 2002; Donahue et al., 1991).

Actualmente se encuentran relictos de este pino, con poblaciones aisladas, árboles solitarios o en pequeños grupos diseminados a lo largo de su distribución natural, particularmente en su zona norte, en los límites estatales de Veracruz y Puebla (Rodríguez y Arteaga, 2005), por lo que el gobierno mexicano considera a esta conífera en la NOM 059-ECOL-2001 en la categoría de protección especial desde 1994 (SEMARNAT, 2010).

La fragmentación en poblaciones pequeñas y la 
baja densidad de arbolado adulto generan polinización deficiente, dispersión y flujo de genes reducido entre poblaciones y un alto grado de autofecundación (Rajora y Mosseler, 2001). El aislamiento geográfico puede reducir la variabilidad genética dentro de las poblaciones al reducirse el flujo genético y propiciar diferenciación morfológica entre ellas (Juárez-Agís et al., 2006). El alto grado de fragmentación del hábitat en la mayoría de las poblaciones explica la diversidad genética reducida en las poblaciones de la especie (Del Castillo et al., 2009).

Usualmente las poblaciones ubicadas en los márgenes de la distribución natural de las coníferas están fragmentadas y aisladas de otras (Mosseler et al., 2000), con capacidad reproductiva baja debido a la producción reducida de semilla viable (Flores-López et al., 2005; 2012). Para conocer el estado reproductivo de las coníferas se ha utilizado el análisis de la producción de semillas por cono (López-Upton y Donahue, 1995; Morales-Velázquez et al., 2010; Owens y Fernando, 2007). El estudio de la variación morfológica de conos y de la proporción de óvulos abortados, semillas llenas, vanas y plagadas, y la germinación de las semillas ayuda a diagnosticar la viabilidad de poblaciones naturales y de unidades productoras de semillas (Bramlett et al., 1977; Mosseler et al., 2000).

La diversidad de ambientes donde crece P. chiapensis y el diferente tamaño poblacional de sus relictos supone una variable, aunque reducida producción y germinación de semilla entre poblaciones. El objetivo del presente estudio fue determinar la capacidad de producción de semillas y germinativa de siete poblaciones septentrionales de $P$. chiapensis en relictos en los estados de Puebla y Veracruz, México y asociar los valores a la densidad poblacional, elevación y algunas variables ambientales.

\section{MATERIALES Y MÉTODOS}

\section{Área de estudio}

Durante agosto y septiembre de 2016 se realizó la recolecta de conos en siete rodales de $P$. chiapensis en los estados de Puebla y Veracruz, México (Figura 1). Las poblaciones se localizan en un intervalo altitudinal de 664 a $1703 \mathrm{msnm}$. En total se seleccionaron 125 individuos dominantes, de 15 a 22 individuos por población (Cuadro 1). Para reducir la probabilidad de parentesco y abarcar la mayor parte de cada localidad, los árboles seleccionados se localizaron entre sí a una distancia mayor de $50 \mathrm{~m}$. En cada relicto se determinó la densidad de población de árboles al contar el número de individuos de $P$. chiapensis con diámetro normal superior a $5 \mathrm{~cm}$ rodeando al árbol seleccionado en una superficie de $1000 \mathrm{~m}^{2}$.

\section{Análisis de conos}

De cada árbol se tomó una muestra de 10 conos para determinar la capacidad reproductiva con la metodología de análisis de conos y producción de semilla (Bramlett et al., 1977; Mosseler et al., 2000). Se registró la longitud y el diámetro del cono cerrado y abierto, una vez secado en invernadero por 15 días a una temperatura de entre 20 y $30{ }^{\circ} \mathrm{C}$. De cada cono se obtuvo el total de semillas llenas, vanas y plagadas y los óvulos abortados (Bramlett et al., 1977). Las escamas fértiles e infértiles se contaron, las primeras producen óvulos fértiles y se ubican en la parte central del cono, mientras que las últimas no presentan óvulos funcionales y se localizan en la base y ápice del cono (Flores-López et al., 2005). Los conos se colocaron en una estufa de secado (Modelo H.102, Laboratorio Riossa, Monterrey, México) a $70{ }^{\circ} \mathrm{C}$ por $48 \mathrm{~h}$ para obtener el peso seco.

De cada cono se determinó:

a) Potencial de semilla $(\mathrm{POT})=$ número de escamas fértiles $\times$ ( 2 óvulos por escama), que es la cantidad límite de semillas producible por cono.

b) Número de semillas desarrolladas, las que completaron su desarrollo de testa = llenas + vanas + plagadas por insectos.

c) Porcentaje de semillas llenas, vanas y plagadas y de óvulos abortados = (número de semillas llenas, vanas o plagadas o número de óvulos abortados/ POT) $\times 100$, la primera se conoce como eficiencia de producción de semillas llenas.

d) La relación del peso de las semillas llenas producidas por cono/peso seco del cono, como una medida de eficiencia reproductiva que refleja la proporción de la energía utilizada en el esfuerzo reproductivo almacenado en la semilla (Mosseler et al., 2000).

\section{Prueba de germinación}

Se utilizó una muestra de 400 semillas por población, mezclando la semilla de los árboles de cada población en cantidades similares. La semilla se distribuyó en cuatro repeticiones de 100 semillas de cada población. La semilla se desinfectó en una solución de hipoclorito de sodio $5 \%$, utilizando solución comercial (Cloralex ${ }^{\circledR}$ ) y se remojó en agua destilada por $24 \mathrm{~h}$ previo a la siembra. La germinación se realizó en cajas de plástico de $33 \times 23 \times$ $10 \mathrm{~cm}$, cada caja representó una unidad experimental. Se utilizó una solución fungicida de Captán en dosis de $3 \mathrm{~g}$ $\mathrm{L}^{-1}$ en la siembra. Las cajas se colocaron en una cámara de germinación (Biotromette Plant Growth Chamber, AB-Line Instruments, Inc., Melrose Park, Illinois, EUA) a temperatura de $26 \pm 2^{\circ} \mathrm{C}$. Diariamente se contaron las semillas germinadas durante 30 días. 


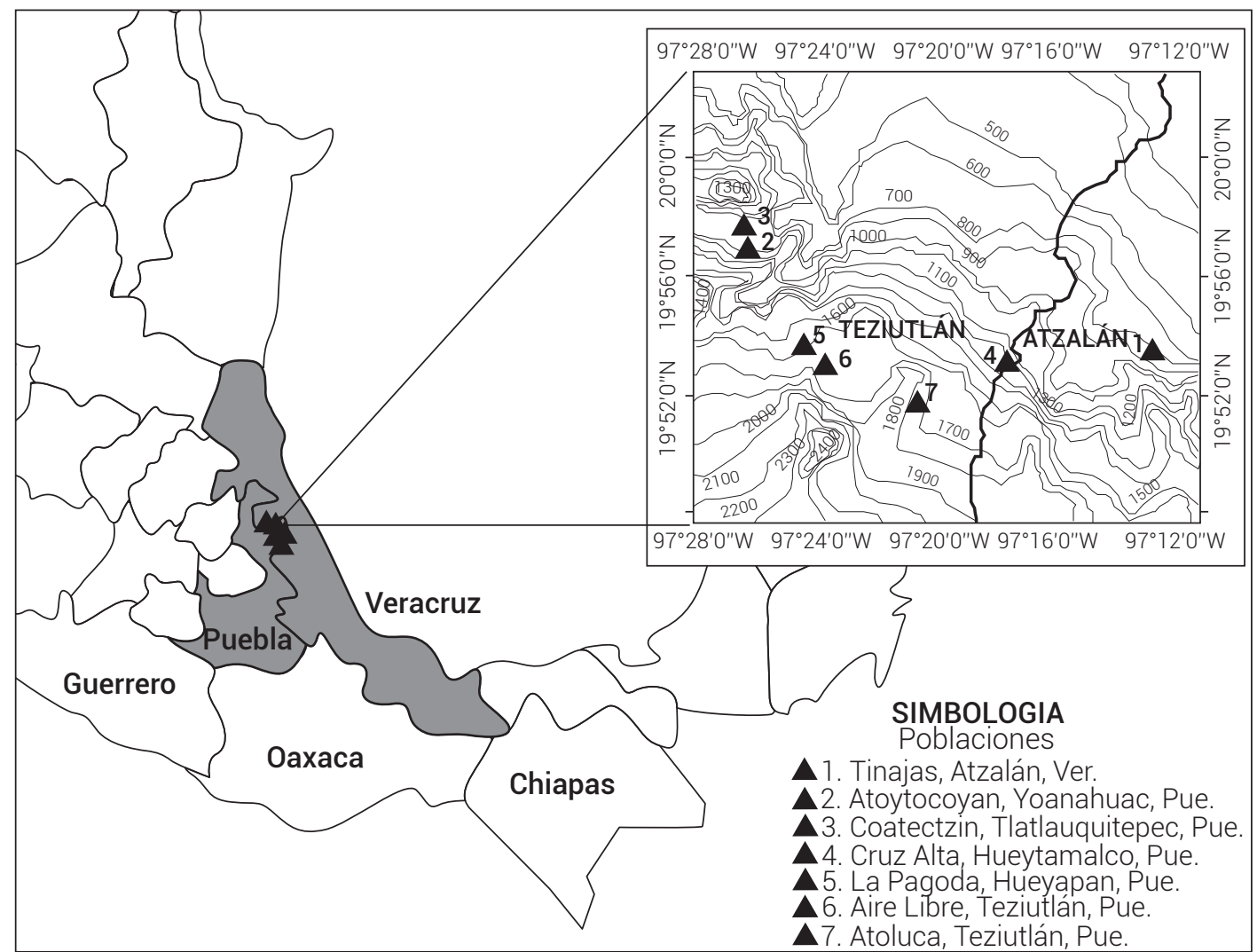

Figura 1. Localización de las poblaciones de Pinus chiapensis en estudio en los estados de Puebla y Veracruz.

Cuadro 1. Datos geográficos y climáticos de los lugares de origen de las poblaciones de Pinus chiapensis (Martínez) Andresen, número de árboles muestreados $(\mathrm{N})$ y densidad de árboles por localidad.

\begin{tabular}{|c|c|c|c|c|c|c|c|}
\hline Localidad, Municipio, Estado & Lat. $\mathrm{N}^{+}$ & Long. $\mathrm{O}$ & Altitud (msnm) & Temp. ${ }^{+\dagger}\left({ }^{\circ} \mathrm{C}\right)$ & $\mathrm{PP}(\mathrm{mm})$ & $N$ & $\begin{array}{c}\text { Densidad } \\
(\text { árboles ha-1) }\end{array}$ \\
\hline Tinajas, Atzalan, Ver. & $19^{\circ} 53^{\prime} 30.7^{\prime \prime}$ & $97^{\circ} 12^{\prime} 53.2^{\prime \prime}$ & 664 & 21.2 & 2676 & 17 & 139 \\
\hline Atotocoyan, Yaonáhuac, Pue. & $19^{\circ} 57^{\prime} 02.6^{\prime \prime}$ & $97^{\circ} 26^{\prime} 24.9^{\prime \prime}$ & 773 & 20.4 & 3427 & 17 & 96 \\
\hline Coatectzin, Tlatlauquitepec, Pue. & $19^{\circ} 57^{\prime} 45.2^{\prime \prime}$ & $97^{\circ} 26^{\prime} 31.2^{\prime \prime}$ & 778 & 20.4 & 3446 & 19 & 100 \\
\hline Cruz Alta, Hueytamalco, Pue. & $19^{\circ} 53^{\prime} 16.2^{\prime \prime}$ & $97^{\circ} 17^{\prime} 46.7^{\prime \prime}$ & 1262 & 17.7 & 2657 & 15 & 123 \\
\hline La Pagoda, Hueyapan, Pue. & $19^{\circ} 53^{\prime} 56.7^{\prime \prime}$ & $97^{\circ} 24^{\prime} 38.9^{\prime \prime}$ & 1568 & 15.9 & 2287 & 22 & 136 \\
\hline Aire Libre, Teziutlán, Pue. & $19^{\circ} 53^{\prime} 02.8^{\prime \prime}$ & $97^{\circ} 23^{\prime} 48.5^{\prime \prime}$ & 1600 & 15.8 & 2170 & 19 & 143 \\
\hline Atoluca, Teziutlán, Pue. & $19^{\circ} 51^{\prime} 47.3^{\prime \prime}$ & $97^{\circ} 20^{\prime} 47.3^{\prime \prime}$ & 1703 & 15.4 & 1881 & 16 & 44 \\
\hline
\end{tabular}

${ }^{\dagger}$ Parte central de cada localidad determinada con un posicionador geográfico con Datum WGS84. ${ }^{+\top T e m p e r a t u r a ~ y ~ p r e c i p i t a c i o ́ n ~ m e d i a ~ a n u a l ~(P P) ~}$ a través de Worldclim. 'Densidad de árboles de P. chiapensis.

Para cada localidad muestreada se determinó la capacidad germinativa o porcentaje final de germinación, el valor pico, que es el valor máximo de la sumatoria del porcentaje de germinación dividido por el número de días en la evaluación y el número de días para alcanzar el 50\% de la germinación total lograda, ambas representan la velocidad de germinación; además, se determinó el valor germinativo, que es el valor pico multiplicado por la germinación promedio (Czabator, 1962; Kolotelo et al., 2001).

\section{Análisis estadístico}

Las características de conos y semillas se analizaron con el procedimiento MIXED del SAS ${ }^{\circledR}$, considerando como efecto fijo las poblaciones y aleatorio los árboles (Littell et al., 2016) con el modelo:

$$
Y_{i j k}=\mu+P_{i}+A_{j(i)}+e_{i j k}
$$


Cuadro 2. Componentes de varianza estimados para las características de conos y semillas de poblaciones de Pinus chiapensis de Puebla y Veracruz.

\begin{tabular}{|c|c|c|c|c|}
\hline \multirow{2}{*}{ Características } & \multicolumn{3}{|c|}{ Componentes de varianza (\%) } & \multirow{2}{*}{ Varianza total } \\
\hline & Población & Árbol/población & Error & \\
\hline Longitud de cono & $46.0 *$ & $36.9 *$ & 17.1 & 400.05 \\
\hline Ancho de cono cerrado & $17.5 *$ & $24.6 *$ & 57.9 & 21.65 \\
\hline Ancho de cono abierto & $58.2 *$ & $20.6 *$ & 21.1 & 63.76 \\
\hline Peso seco del cono (PSC) & $51.9 *$ & $33.1 *$ & 15.0 & 16.05 \\
\hline Potencial de semillas & $30.5 *$ & $35.1 *$ & 34.4 & 385.41 \\
\hline Semilla desarrollada \% & $45.3 *$ & $26.7 \star$ & 28.0 & 666.96 \\
\hline Peso total de semillas llenas (PS) & $36.7 *$ & $30.9 *$ & 32.4 & 0.0704 \\
\hline Peso unitario de semilla & $9.1 *$ & $15.7 *$ & 75.2 & $4.52 \mathrm{E}^{-05}$ \\
\hline Relación PS/PSC & $18.4 *$ & $41.8 *$ & 39.8 & 435.10 \\
\hline Proporción de semillas llenas ${ }^{\dagger}$ & $4.1 *$ & $40.9 *$ & 55.0 & 218.86 \\
\hline Proporción de semillas vanas & $6.8 *$ & $37.8^{\star}$ & 55.4 & 252.22 \\
\hline Proporción de semillas plagadas & $13.9 *$ & $26.3 *$ & 59.8 & 4.719 \\
\hline $\begin{array}{l}\text { Proporción de óvulos abortados } \\
\text { Promedio }\end{array}$ & $\begin{array}{l}16.5 * \\
27.3\end{array}$ & $\begin{array}{l}29.4 * \\
30.8\end{array}$ & $\begin{array}{l}54.1 \\
41.9\end{array}$ & 450.63 \\
\hline
\end{tabular}

*Significativos con $\mathrm{P} \leq 0.05$. ${ }^{\dagger}$ Eficiencia de producción de semilla llena.

Dónde: $Y_{i j k}$ es la observación del k-ésimo cono del j-ésimo árbol de la i-ésima población, $\mu$ es la media poblacional, $P_{i}$ es el efecto de la i-ésima población, $A_{j(i)}$ es el efecto del j-ésimo árbol dentro de población y $e_{i j k}$ es el error experimental de muestreo por el cono analizado. La distribución de la muestra resultó normal en las variables. Con la opción LSMEANS se obtuvieron las medias ajustadas, debido al desbalance en el número de árboles muestreados en cada población. Para determinar la proporción de la variación a nivel población, entre y dentro de árboles en las variables de cono y semilla todos los efectos se consideraron aleatorios y se analizó con el mismo modelo.

Para los datos de germinación se utilizó el modelo:

$$
Y_{i j}=\mu+R_{i}+P_{j}+e_{i j}
$$

Dónde $Y_{i j}$ es el valor de la característica de la j-ésima población en el i-ésimo bloque, $\mu$ es el efecto de la media general, $R_{i}$ es el efecto aleatorio de la i-ésima repetición, $P_{j}$ es el efecto fijo del j-ésima población $(1,2, \ldots 7)$ y $e_{i j}$ es el error experimental. Previamente, los datos de la capacidad germinativa se transformaron con la función arcoseno de la raíz cuadrada de $p(\theta=\operatorname{arcoseno} \sqrt{ } p), \theta$ donde es el dato transformado y $p$ es la capacidad germinativa sin transformar), para normalizar la distribución (Sokal y Rohlf, 1981). La prueba de homogeneidad de varianza indicó que las muestras son homocedásticas.

Para determinar la existencia de un patrón de variación morfológica entre las poblaciones de Pinus chiapensis se calculó el coeficiente de correlación de Pearson entre los valores promedio de las características de conos, semillas y germinación contra la altitud de origen de cada población, variables climáticas y la densidad de los rodales estudiados.

\section{RESULTADOS}

\section{Características reproductivas}

Se encontraron diferencias $(P \leq 0.05)$ entre poblaciones y dentro de cada población para las variables de conos y semillas de P. chiapensis. La mayor aportación a la varianza total (41.9\%) correspondió al error de muestreo; así, en seis de las 13 características evaluadas la mayor varianza fue entre los conos, particularmente los porcentajes de producción de los tres tipos de semilla y los óvulos abortados, así como el diámetro del cono y el peso promedio de una semilla llena. Las poblaciones presentaron el mayor aporte a la varianza en cinco de las 13 características evaluadas, más notorio fue en las relativas al tamaño de cono y peso de las semillas llenas por cono (Cuadro 2).

El largo y ancho de cono promedio de las poblaciones fue de 95.9 y 28.7 mm, respectivamente. La población Aire Libre tuvo los valores más altos (120.4 y 31.4 mm), mientras que en la población Coatectzin se encontraron los conos de menor longitud $(83.7 \mathrm{~mm})$ y en la población Cruz Alta los más angostos (26.1 mm) (Cuadro 3). 
Cuadro 3. Diferencias entre valores promedio ( \pm error estándar) de características reproductivas de conos y semillas de poblaciones de Pinus chiapensis de Puebla y Veracruz.

\begin{tabular}{|c|c|c|c|c|c|c|c|}
\hline \multirow{2}{*}{ Variables } & \multicolumn{7}{|c|}{ Poblaciones $^{\dagger}$} \\
\hline & Tinajas & Atotocoyan & Coatectzin & Cruz Alta & La Pagoda & Aire Libre & Atoluca \\
\hline Longitud de cono (mm) & $86.4 \pm 0.6 d$ & $86.3 \pm 0.6 d$ & $83.7 \pm 0.6 \mathrm{e}$ & $101.5 \pm 0.7 \mathrm{c}$ & $106.3 \pm 0.6 b$ & $120.4 \pm 0.6 a$ & $86.8 \pm 0.7 d$ \\
\hline Ancho de cono cerrado (mm) & $27.0 \pm 0.2 c$ & $26.1 \pm 0.3 d$ & $27.3 \pm 0.3 c$ & $29.2 \pm 0.3 b$ & $31.0 \pm 0.2 \mathrm{a}$ & $31.4 \pm 0.3 a$ & $29.0 \pm 0.3 b$ \\
\hline Ancho de cono abierto (mm) & $38.6 \pm 0.3 c$ & $33.6 \pm 0.3 e$ & $35.1 \pm 0.3 d$ & $40.8 \pm 0.3 b$ & $40.9 \pm 0.3 b$ & $52.7 \pm 0.3 a$ & $40.8 \pm 0.3 b$ \\
\hline Peso seco de cono (PSC) (g) & $10.4 \pm 0.1 d$ & $8.2 \pm 0.1 \mathrm{e}$ & $7.8 \pm 0.1 \mathrm{e}$ & $12.3 \pm 0.1 \mathrm{c}$ & $13.2 \pm 0.1 b$ & $16.1 \pm 0.1 \mathrm{a}$ & $10.2 \pm 0.1 d$ \\
\hline Potencial de semilla & $96.6 \pm 0.9 c$ & $75.9 \pm 0.9 f$ & $75.9 \pm 0.8 f$ & $91.2 \pm 0.9 d$ & $98.7 \pm 0.8 b$ & $102.8 \pm 0.8 a$ & $81.1 \pm 0.9 \mathrm{e}$ \\
\hline Semilla desarrollada (\%) & $52.4 \pm 1.1 \mathrm{a}$ & $33.2 \pm 1.1 \mathrm{c}$ & $37.5 \pm 1.0 \mathrm{bc}$ & $37.8 \pm 1.2 b$ & $55.7 \pm 1.0 \mathrm{a}$ & $56.5 \pm 1.0 \mathrm{a}$ & $13.8 \pm 1.1 \mathrm{~d}$ \\
\hline $\begin{array}{l}\text { Peso total de semillas llenas } \\
\text { (PS) (mg) }\end{array}$ & $300 \pm 12 c$ & $125 \pm 12 \mathrm{e}$ & $181 \pm 11 d$ & $273 \pm 12 c$ & $538 \pm 10$ a & $516 \pm 11 b$ & $97 \pm 12 f$ \\
\hline Peso unitario de semilla (mg) & $15.2 \pm 0.2 d$ & $14.4 \pm 0.3 \mathrm{e}$ & $15.3 \pm 0.3 d$ & $19.1 \pm 0.2 b$ & $18.8 \pm 0.2 b$ & $20.9 \pm 0.2 a$ & $17.8 \pm 0.3 c$ \\
\hline Relación PS/PSC $\left(\mathrm{mg} \mathrm{g}^{-1}\right)^{++}$ & $28.8 \pm 1.0 \mathrm{~b}$ & $15.2 \pm 1.1 \mathrm{~d}$ & $23.2 \pm 1.0 \mathrm{c}$ & $22.2 \pm 1.1 \mathrm{c}$ & $40.8 \pm 0.9 a$ & $32.0 \pm 1.0 \mathrm{~b}$ & $9.5 \pm 1.1 \mathrm{e}$ \\
\hline Proporción semillas llenas (\%) & $20.4 \pm 0.6 c$ & $11.4 \pm 0.7 \mathrm{e}$ & $15.6 \pm 0.6 d$ & $15.7 \pm 0.7 d$ & $29.0 \pm 0.6 a$ & $24.0 \pm 0.6 b$ & $6.7 \pm 0.7 f$ \\
\hline Proporción semillas vanas (\%) & $28.6 \pm 0.9 b$ & $21.3 \pm 0.9 d$ & $21.7 \pm 0.8 d$ & $21.9 \pm 0.9 d$ & $25.9 \pm 0.8 c$ & $32.3 \pm 0.8 \mathrm{a}$ & $6.9 \pm 1.0 \mathrm{e}$ \\
\hline $\begin{array}{l}\text { Proporción semillas } \\
\text { plagadas (\%) }\end{array}$ & $3.6 \pm 0.1 \mathrm{a}$ & $0.5 \pm 0.1 c$ & $0.4 \pm 0.1 c$ & $0.2 \pm 0.1 \mathrm{~d}$ & $0.8 \pm 0.1 b$ & $0.2 \pm 0.1 d$ & $0.2 \pm 0.1 \mathrm{~d}$ \\
\hline Proporción óvulos abortados (\%) & $47.4 \pm 1.1 \mathrm{~d}$ & $66.8 \pm 1.1 b$ & $62.3 \pm 1.0 \mathrm{c}$ & $62.2 \pm 1.2 \mathrm{c}$ & $44.3 \pm 1.0 \mathrm{e}$ & $43.5 \pm 1.0 \mathrm{e}$ & $86.2 \pm 1.1 \mathrm{a}$ \\
\hline
\end{tabular}

Medias con letras iguales en las hileras no son estadísticamente diferentes $(\mathrm{P} \leq 0.05) .{ }^{\dagger}$ Ordenadas de menor a mayor elevación. ${ }^{\dagger+}$ Eficiencia en la producción de semilla.

El número potencial promedio de semillas por cono de P. chiapensis fue de 89, con valores promedio extremos de 76 (Atotocoyan y Coatectzin) y 103 (Aire Libre). Los promedios de óvulos abortados, semillas llenas, vanas y plagadas fueron $59,17.5,22.6$ y $0.9 \%$ del potencial de semillas, respectivamente. A pesar de las diferencias entre poblaciones, en general, la eficiencia de semillas resultó muy baja ( $17.5 \%$ en promedio, < $30 \%$ considerando el valor de cada población), Atoluca con la menor proporción (6.7 \%) y La Pagoda con la mayor (29 \%). Se presentó un promedio de 16 semillas llenas por cono, que varía entre poblaciones de 5 a 29 semillas por cono (98.7 semillas potenciales $\times 0.29$ de eficiencia; Cuadro 3 ).

Los valores de óvulos abortados variaron de $43.5 \%$ en Aire Libre a $86.2 \%$ en Atoluca. El peso promedio de una semilla de P. chiapensis fue $17.4 \mathrm{mg}$ y varió de 14.4 (Atotocoyan) a $20.9 \mathrm{mg}$ (Aire Libre), por lo que en un kg habría entre 47,850 y 69,440 semillas llenas. En promedio se obtuvo $24.5 \mathrm{mg}$ de semilla por g de estróbilo maduro, de $9.5 \mathrm{mg} \mathrm{g}^{-1}$ en Atoluca a $40.8 \mathrm{mg} \mathrm{g}^{-1}$ en La Pagoda (Cuadro 3).

\section{Prueba de germinación}

Se encontró una variación significativa $(P \leq 0.05)$ entre poblaciones en las variables evaluadas. El valor promedio de germinación fue de $72 \%$, con valores extremos de 50 a $87 \%$. En promedio fueron necesarios 14 días para alcanzar 50 \% de la germinación total, seis días de diferencia entre poblaciones extremas (Cuadro 4). El valor pico promedio fue de $3.74 \%$ por día, la población de mayor valor fue del doble que la de menor valor (2.26 a $4.44 \%$ ). El valor germinativo promedio fue de 9.74 , variable entre poblaciones, ya que fue de tres veces entre el menor y mayor valor (3.9 a 12.9), sin relación con las variables ambientales o de altitud. Las semillas de las poblaciones con menores porcentajes de germinación tuvieron un proceso más lento (Figura 2; Cuadro 4).

\section{Análisis de correlación}

La altitud se asoció positivamente con el ancho del cono cerrado y con el peso de la semilla, y ligeramente con el peso seco del cono. La temperatura se asoció negativamente con estas mismas variables. Tanto la 
altitud como la temperatura correlacionaron de forma negativa con la proporción de semillas vanas sobre las desarrolladas, la primera además, de manera positiva con la precipitación. La precipitación se asoció negativamente con el ancho y con el peso de los conos y de la semilla. La densidad de los árboles se asoció positivamente con las tres variables de cantidad de semillas en producción y con el peso total de semillas por cono, y negativamente con óvulos abortados y semillas vanas (Cuadro 5).

La capacidad germinativa no seasoció significativamente con las variable ambientales o de densidad de árboles, aunque la velocidad de germinación lo hizo positivamente con la altitud, y por tanto, de manera negativa con la temperatura; esto es, la velocidad de germinación fue menor al disminuir la temperatura del sitio de origen
(Cuadro 5).

\section{DISCUSIÓN}

La variación observada entre poblaciones de Pinus chiapensis en las características de cono también ha sido reportada para otras especies de pino (IglesiasAndreu et al., 2012; Martínez, 1998; Sáenz-Romero et al., 2012). La variación en el largo y ancho de cono en pinos ha sido relacionada con factores ambientales (LópezUpton y Donahue, 1995). En este estudio, con excepción de la población del margen altitudinal superior, Atoluca, las poblaciones de mayor altitud mostraron los conos más grandes y una mayor producción de semilla, La Pagoda con 29 y Aire Libre con 25 semillas llenas por cono; en contraparte, en las poblaciones de menor altitud, Tinajas,

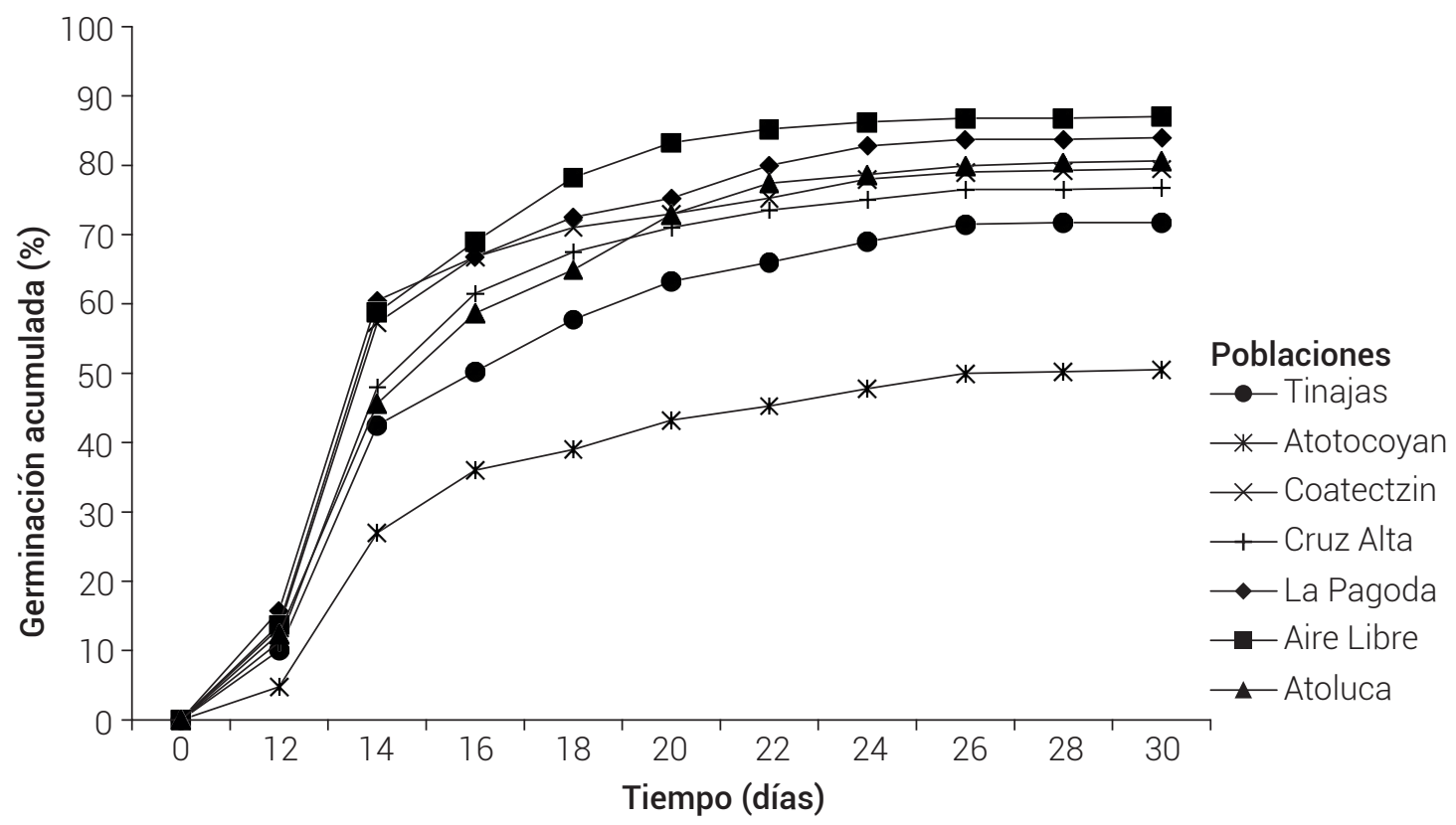

Figura 2. Curva de germinación acumulada de lotes de semillas de siete poblaciones de Pinus chiapensis de Puebla y Veracruz.

Cuadro 4. Diferencias entre valores promedio ( \pm error estándar) de características germinativas de semillas de poblaciones de Pinus chiapensis de Puebla y Veracruz.

\begin{tabular}{lcccc}
\hline Población $^{+}$ & Capacidad germinativa (\%) & Días a la germinación al 50\% & Valor pico (\% día $\left.{ }^{-1}\right)$ & Valor germinativo \\
\hline Tinajas & $71.75 \pm 2.69 \mathrm{~d}$ & $18.50 \pm 0.85 \mathrm{~d}$ & $3.24 \pm 0.18 \mathrm{~d}$ & $7.74 \pm 0.78 \mathrm{~d}$ \\
Atotocoyan & $50.50 \pm 2.69 \mathrm{e}$ & $17.00 \pm 0.85 \mathrm{c}$ & $2.26 \pm 0.18 \mathrm{e}$ & $3.82 \pm 0.78 \mathrm{e}$ \\
Coatectzin & $79.50 \pm 2.69 \mathrm{bc}$ & $15.00 \pm 0.85 \mathrm{~b}$ & $4.20 \pm 0.18 \mathrm{ab}$ & $11.22 \pm 0.78 \mathrm{bc}$ \\
Cruz Alta & $76.75 \pm 2.69 \mathrm{c}$ & $14.50 \pm 0.85 \mathrm{~b}$ & $3.85 \pm 0.18 \mathrm{c}$ & $9.94 \pm 0.78 \mathrm{c}$ \\
La Pagoda & $84.00 \pm 2.69 \mathrm{ab}$ & $14.00 \pm 0.85 \mathrm{ab}$ & $4.29 \pm 0.18 \mathrm{ab}$ & $12.05 \pm 0.78 \mathrm{ab}$ \\
Aire Libre & $87.00 \pm 2.69 \mathrm{a}$ & $12.50 \pm 0.85 \mathrm{a}$ & $4.44 \pm 0.18 \mathrm{a}$ & $12.86 \pm 0.78 \mathrm{a}$ \\
Atoluca & $80.75 \pm 2.69 \mathrm{bc}$ & $15.00 \pm 0.85 \mathrm{~b}$ & $3.93 \pm 0.18 \mathrm{bc}$ & $10.58 \pm 0.78 \mathrm{bc}$ \\
\hline Fordenadas de menor a mayor elevación. Medias con letras igales en cada
\end{tabular}


Cuadro 5. Coeficientes de correlación entre variables ambientales y variables morfológicas de conos, semillas y germinación de Pinus chiapensis de poblaciones de Puebla y Veracruz.

\begin{tabular}{|c|c|c|c|c|}
\hline Variable morfológica & Altitud & Temperatura & Precipitación & Densidad \\
\hline Longitud de cono & 0.64 & -0.66 & -0.52 & 0.58 \\
\hline Ancho de cono cerrado & $0.88 * *$ & $-0.89 * \star$ & $-0.79 *$ & 0.30 \\
\hline Ancho de cono abierto & $0.68^{+}$ & $-0.68^{+}$ & $-0.71^{+}$ & 0.38 \\
\hline Peso seco de cono (PSC) & $0.69^{+}$ & $-0.69^{+}$ & $-0.69^{+}$ & 0.56 \\
\hline Potencial de producción de semilla & 0.40 & -0.40 & -0.60 & $0.76 *$ \\
\hline Semilla desarrollada & 0.05 & -0.06 & -0.20 & $0.94 *$ \\
\hline Peso total de semillas llenas (PS) & 0.43 & -0.44 & -0.43 & $0.79 *$ \\
\hline Peso unitario de semilla & $0.85 * *$ & $-0.86 * *$ & $-0.74^{+}$ & 0.29 \\
\hline Relación PS/PSC & 0.09 & -0.11 & -0.13 & $0.87 * *$ \\
\hline Proporción de semillas llenas & 0.24 & -0.26 & -0.31 & $0.86 * *$ \\
\hline Proporción de semillas vanas & -0.24 & 0.22 & 0.16 & $-0.96 * *$ \\
\hline Proporción de óvulos abortados & 0.09 & -0.07 & 0.01 & $-0.97 \star \star *$ \\
\hline Proporción semillas vanas/desarrolladas & $-0.74 *$ & $0.74 *$ & $0.85 * *$ & -0.16 \\
\hline Capacidad germinativa & 0.66 & -0.66 & -0.66 & 0.18 \\
\hline Días a la germinación al 50 \% & $-0.78 *$ & $0.79 *$ & 0.45 & -0.11 \\
\hline Valor pico & 0.58 & -0.58 & -0.46 & 0.27 \\
\hline Valor germinativo & 0.63 & -0.64 & -0.54 & 0.25 \\
\hline
\end{tabular}

${ }_{+}, * \mathrm{y} * *$ : significativo con $\mathrm{P} \leq 0.10, \mathrm{P} \leq 0.05 \mathrm{y} \mathrm{P} \leq 0.01$. $^{\dagger}$ Eficiencia de producción de semilla llena.

Atotocoyan y Coatectzin se obtuvieron 20, 9 y 12 semillas llenas por cono, respectivamente. La producción de semilla fue inferior a la de poblaciones de Chiapas, de 43 a 79 semillas llenas por cono, con una eficiencia de 44 a 59 \% (Martínez, 1998), mientras que en este estudio fue de 6.7 a $29 \%$. Lo anterior evidencia que en las poblaciones muestreadas de Puebla-Veracruz existen problemas reproductivos en Pinus chiapensis.

Se requiere de 1860 conos (con 29 semillas en promedio) a 10,300 (con 6 semillas) para reunir $1 \mathrm{~kg}$ de semilla de la especie entre las poblaciones extremas, lo que proporciona una idea de la magnitud del trabajo necesario para cubrir una meta de plantación. Esta variación se atribuye a las diferencias de densidad poblacional en las cuales crece la especie. Hernández y Eguiluz (1986) concluyeron que el patrón de variación en las características morfológicas de cono de P. chiapensis en poblaciones de Oaxaca y Chiapas es discontinuo y ecotípico.

Los valores bajos de eficiencia de producción de semilla y altos porcentajes de semilla vana y óvulos abortados indican problemas de depresión endogámica, común en coníferas con poblaciones reducidas, ejemplos de ello se presentan en Pinus hartwegii Lindl. (Iglesias-Andreu et al., 2012), P. pinceana Gordon (Quiroz-Vázquez et al., 2017), P. leiophylla Schltdl. et Cham. (Morales-Velázquez et al.,
2010), Picea mexicana Martínez (Flores-López et al., 2005) y P. martinezii T. F. Patterson (Flores-López et al., 2012). En poblaciones reducidas existe alta probabilidad de cruzas entre individuos emparentados, o incluso autofecundación, lo que genera la acción de genes deletéreos que provocan nulo o lento desarrollo del embrión, y por consiguiente la formación de semilla vana o de baja calidad (Owens y Fernando, 2007). En poblaciones pequeñas y con baja densidad de árboles la cantidad y la calidad de polen son considerablemente bajos (Mosseler et al., 2000). Las poblaciones muestreadas de $P$. chiapensis variaron de 3.2 a 39 ha, con promedio de 19 ha, lo que representa un tamaño muy reducido para una conífera, y explica la alta cantidad de óvulos abortados de este pino en esta región.

A pesar de lo señalado, la capacidad germinativa en las semillas fue mayor de 50\%. Estos valores son similares a los porcentajes reportados por Martínez (1998) para poblaciones de Chiapas. Esta variable no se asoció con los niveles de densidad de los árboles para cada población, por lo que los efectos de la endogamia sólo se reflejaron en la baja producción de semilla llena.

En el análisis de correlación se encontró una asociación positiva de las características morfológicas de cono con la altitud de origen de las poblaciones evaluadas, lo que indica diferencias adaptativas entre éstas, así se ha determinado 
también en otras especies de pinos mexicanos al asociar estas variables con la elevación del sitio de recolecta (López-Toledo et al., 2017; Viveros-Viveros et al., 2013). Algunas poblaciones de coníferas muestran adaptaciones fisiológicas a sus ambientes particulares (Rehfeldt, 1983), situación que ha propiciado su diferenciación; tal es el caso de pinos mexicanos como Pinus patula Schiede ex Schltdl. et Cham. y P. pseudostrobus Lindl., que crecen en ambientes diferentes y han tenido que adaptarse a factores ecológicos contrastantes, lo cual se refleja en el crecimiento de sus plántulas (Sáenz-Romero et al., 2011; 2012).

Independientemente de la altitud, la densidad tuvo un mayor papel en la producción de semillas y en el porcentaje de óvulos abortados, como se reveló en la correlación con esas variables, donde poblaciones con muchos árboles por hectárea presentaron mayor producción de semilla con menos óvulos abortados, lo cual implica una baja producción de polen, además de la acción de procesos de endogamia en poblaciones de menor densidad (Cain y Shelton, 2000). La baja densidad de árboles adultos y aislados en algunas de las poblaciones hace suponer que el éxito reproductivo y la germinación de la semilla son relativamente bajos en poblaciones fragmentadas, como lo muestran estudios de producción y germinación de semillas de coníferas (Juárez-Agís et al., 2006; Mápula et al., 2008). En general, las tres poblaciones con más de 135 árboles de P. chiapensis resultaron con mejores valores de producción de semillas llenas y con mayor peso, aunque en este caso la germinación de P. chiapensis resultó, salvo en una población, mayor de $75 \%$, lo cual es un valor adecuado. Ésto lleva a considerar estrategias de conservación in situ y ex situ, mediante el desarrollo de acciones para contrarrestar problemas de endogamia en dichas poblaciones, con introducción e intercambio de material genético entre ellas para ampliar la base genética y favorecer el cruzamiento de individuos no emparentados en las siguientes generaciones.

\section{CONCLUSIONES}

Existe diferenciación en las poblaciones de Puebla y Veracruz de P. chiapensis en características morfológicas de cono, producción de semilla y germinación. A pesar de que la producción de semilla es reducida, el porcentaje de germinación es aceptable. La divergencia entre poblaciones en las características morfológicas de los conos está asociada con la elevación de los sitios de origen, se presentan mayores dimensiones en conos y semillas, velocidad y porcentaje de germinación en poblaciones de sitios de mayor altitud. La densidad de las poblaciones de Pinus chiapensis es importante en el éxito de las características reproductivas de la especie,
135 árboles por hectárea serían necesarios para obtener valores adecuados en la producción de semillas llenas.

\section{BIBLIOGRAFÍA}

Bramlett D. L., E. W. Belcher, G. L. DeBarr, G. D. Hertel, R. P. Karrfalt, C. W. Lantz, ... and H. O. Yates (1977) Cone Analysis of Southern Pines: A Guidebook. General Technical Report SE-13. Southeastern Forest Experiment Station, United States Department of Agriculture. Asheville, North Carolina and Southeastern Area, State and Private Forest, Atlanta, Georgia, USA. 28 p.

Cain M. D. and M. G. Shelton (2000) Revisiting the relationship between common weather variables and loblolly-shortleaf pine seed crops in natural stands. New Forests 19:187-204, https://doi. org/10.1023/A:1006624907975

Czabator F. J. (1962) Germination value: an index combining speed and completeness of pine seed germination. Forest Science 8:386396, https://doi.org/10.1093/forestscience/8.4.386

Del Castillo R. F. and S. Acosta T. (2002) Ethnobotanical notes on Pinus strobus var. chiapensis. Anales del Instituto de Biología, Serie Botánica 73:319-327.

Del Castillo R. F., S Trujillo A. and C. Sáenz-Romero (2009) Pinus chiapensis, a keystone species: genetics, ecology and conservation. Forest Ecology and Management 257:2201-2208, https://doi. org/10.1016/j.foreco.2009.03.004

Donahue J. K., W. S. Dvorak and E. A. Gutierrez (1991) The Distribution, Ecology, and Gene Conservation of Pinus ayacahuite and Pinus chiapensis in Mexico and Central America. CAMCORE Bulletin on Tropical Forestry No. 8. College of Forest Resources, North Carolina State University. Raleigh, North Carolina, USA. 56 p.

Flores-López C., J. López-Upton y J. J. Vargas-Hernández (2005) Indicadores reproductivos en poblaciones naturales de Picea mexicana Martínez. Agrociencia 39:117-126.

Flores-López C., C. G. Geada-López., J. López-Upton y E. López-Ramírez (2012) Producción de semilla e indicadores reproductivos en poblaciones naturales de Picea martinezii T. F. Patterson. Revista Forestal Baracoa 31:49-58.

Hernández G. J. J. y T. Eguiluz P. (1986) Variación morfológica de acículas, conos y semillas de Pinus chiapensis (Martínez) Andresen de Oaxaca y Chiapas. Revista Chapingo 75:25-33.

Iglesias-Andreu L. G., L. Y. Solís-Ramos y H. Viveros-Viveros (2012) Variación morfométrica en dos poblaciones naturales de Pinus hartwegii. Lindl. del estado de Veracruz. Phyton 81:239-246.

Juárez-Agís A., J. López-Upton, J. J. Vargas-Hernández y C. Sáenz-Romero (2006) Variación geográfica en la germinación y crecimiento inicial de plántulas de Pseudotsuga menziesii de México. Agrociencia 40:783-792

Kolotelo D., E. van Steenis, M. Peterson, R. Bennett, D. Trotter and J. Dennis (2001) Seed Handling Guidebook. Ministry of Forests, Tree Improvement Branch. British Columbia, Canada. $106 \mathrm{p}$

Littell R. C., G. A. Milliken, W. W. Stroup, R. D. Wolfinger and O. Schabenberger (2016) SAS System for Mixed Models. 2nd edition. Cary, North Carolina, USA. $814 \mathrm{p}$

López-Toledo L., M. Heredia-Hernández, D. Castellanos-Acuña, A. BlancoGarcia and C. Sáenz-Romero (2017) Reproductive investment of Pinus pseudostrobus along an altitudinal gradient in western México: implications of climate change. New Forests 48:467481, https://doi.org/10.1007/s11056-017-9602-8

López-Upton J. and J. K. Donahue (1995) Seed production of Pinus greggii Engelm. in natural stands in Mexico. Tree Planters' Notes 46:86-92.

Mápula L. M., J. López U., J. J. Vargas H. y A. Hernández L. (2008) Germinación y vigor de semillas de Pseudotsuga menziesii de México. Ra Ximhai 4:119-134.

Martínez C. N. (1998) Atributos poblacionales y reproductivos de Pinus chiapensis en Chiapas, México. Anales del Instituto de Biología, Serié Botánica 69:119-134

Morales-Velázquez M. G., C. A. Ramírez-Mandujano, P. Delgado-Valerio y J. López-Upton (2010) Indicadores reproductivos de Pinus leiophylla Schltdl. et Cham. en la cuenca del rio Angulo, Michoacán. Revista Mexicana de Ciencias Forestales 1:31-38, https://doi.org/10.29298/rmcf.v1i2.635 
Mosseler A., J. E. Major, J. D. Simpson, B. Daigle, K. Lange, Y.-S. Park, ... and O. P. Rajora (2000) Indicators of populations viability in red spruce, Picea rubens. I. Reproductive traits and fecundity. Canadian Journal of Botany 78:928-940, https://doi.org/10.1139/b00065

Owens J. N. and D. D. Fernando (2007) Pollination and seed production in western white pine. Canadian Journal of Forest Research 37:260-275, https://doi.org/10.1139/X06-220

Quiroz-Vázquez R. I., J. López-Upton, V. M. Cetina-Alcalá y G. Ángeles-Pérez (2017) Capacidad reproductiva de Pinus pinceana Gordon en el límite sur de su distribución natural. Agrociencia 51:91-104.

Rajora O. P. and A. Mosseler (2001) Challenges and opportunities for conservation of forest genetic resources. Euphytica 118:197212, https://doi.org/10.1023/A:1004150525384

Rehfeldt G. E. (1983) Genetic variability within Douglas-fir populations: implications for tree improvement. Silvae Genetica 32:9-14.

Rodríguez A. M. y B. Arteaga M. (2005) Índice de sitio para Pinus chiapensis (Martínez) Andresen, en los estados de Puebla y Veracruz, México. Revista Chapingo, Serie Ciencias Forestales y del Ambiente 11:39-44.

Sáenz-Romero C., L. F. Ruiz-Talonia, J. Beaulieu, N. M. Sánchez-Vargas and G. E. Rehfeldt (2011) Genetic variation among Pinus patula populations along an altitudinal gradient. Two environment nursery tests. Revista Fitotecnia Mexicana 34:19-25, https:// doi.org/10.35196/rfm.2011.1.19

Sáenz-Romero C. G. Rehfeldt, J. Soto-Correa, S. Aguilar-Aguilar, V. ZamarripaMorales y J. López-Upton (2012) Altitudinal genetic variation among Pinus pseudostrobus populations from Michoacán Mexico. Two location shadehouse test results. Revista Fitotecnia Mexicana 35:111-121, https://doi.org/10.35196/ rfm.2012.2.111

SEMARNAT (2010) Norma Oficial Mexicana NOM-059-ECOL-2001. Protección ambiental-Especies nativas de México de flora y fauna Silvestre. Categorías de riesgo y especificaciones para su inclusión, exclusión o cambio-Lista de especies en riesgo. Diario Oficial de la Federación, 30 de diciembre de 2010. México, D. F. pp:1-78

Sokal R. R. and F. J. Rohlf (1981) Biometry: The Principles of Practice of Statistics in Biological Research. W.H. Freeman and Company. San Francisco, California, USA. 776 p. https://doi. org/10.2307/1378519

Viveros-Viveros H., A. R. Camarillo-Luna, C. Sáenz-Romero y A. AparicioRentería (2013) Variación altitudinal en caracteres morfológicos de Pinus patula en el estado de Oaxaca (México) y su uso en la zonificación. Bosque 34:173-179, http://doi.org/10.4067/ S0717-92002013000200006 
